\title{
Short Call Abstract Author Index
}

Abdeen, M. Short Call Poster 52

Adewoye, A. Short Call Poster 61, Short Call

Poster 63, Short Call Poster 64

Adzick, S. Short Call Poster 46

Agate, L. Short Call Oral 2

Ali, S. 462, Short Call Poster 55

Andrade, C.B. Short Call Poster 51

Anochie, O. 313, Short Call Poster 54

Arosemena, M. Short Call Poster 73, Short Call Poster 75

Babiarz, J.E. 461, 462, Short Call Poster 55 Baloch, Z.W. 461, Short Call Poster 48

Barber, A. Short Call Poster 21

Basolo, F. 335, Short Call Oral 2, Short Call Oral 7

Bassett, R. Short Call Poster 45

Bauer, A.J. Short Call Poster 46

BAZ, O.H. Short Call Poster 19

Bernet, V. 398, Short Call Oral 3

Bianchini, V. Short Call Oral 7

Blanco, N. Short Call Poster 51

Bloise, F.F. Short Call Poster 51

Bo, Z. Short Call Poster 26,

Short Call Poster 27

Bolf, E.L. Short Call Poster 41, Short Call Poster 60

Bonnema, S. Short Call Oral 6

Bornschlegl, S. Short Call Poster 12

Bottici, V. Short Call Oral 2

Brett, C. Short Call Oral 3

Brix, T.H. Short Call Oral 6

Broome, D.T. Short Call Poster 20

Bubnov, A. Short Call Poster 67

Bundele, M.M. Short Call Poster 33

Busaidy, N.L. 389, 394, Oral 32,

Short Call Oral 10, Short Call Oral 9,

Short Call Poster 25,

Short Call Poster 40, Short Call Poster 45

Buzanakov, D. Short Call Poster 67

Cabanillas, M.E. 389, 394, Oral 32, Short Call Oral 10, Short Call Oral 9, Short Call Poster 25,

Short Call Poster 40, Short Call Poster 45

Camacho, J.C. Short Call Poster 62

Canner, J. Short Call Poster 49

Cao, J. Short Call Poster 39

Carr, F. Short Call Poster 41, Short Call Poster 60, Short Call Poster 70

Casler, J. Short Call Oral 3

Castillo, P. Short Call Poster 73, Short Call Poster 75

Caturegli, P. Short Call Oral 1

Chalan, P. Short Call Oral 1

Chazen, R.S. Short Call Poster 37

Cheah, W. Short Call Poster 33
Chelghoume, I. Short Call Poster 19

Chen, C. 176, Short Call Poster 39

Chen, W. Short Call Poster 44, Short Call Poster 50

Cheng, P. Short Call Poster 38

Chernicov, R. Short Call Poster 67

Chin, D.C. Short Call Poster 33

Chinchuk, I. Short Call Poster 67

Chindris, A. Short Call Oral 3

Chua, D.Y. Short Call Poster 33

Ciampi, R. Short Call Oral 2

Clarke, O. Short Call Poster 71

Clayman, G.L. Short Call Poster 65

Cote, D.W. Short Call Poster 34

Cote, G.J. 389, Oral 32, Short Call Poster 25 , Short Call Poster 45

Cvek, U. Short Call Poster 61

Dadu, R. 389, Oral 32, Short Call Oral 10, Short Call Oral 9, Short Call Poster 25, Short Call Poster 45

Dang, H. Short Call Poster 43

Danysh, B. Short Call Poster 40

Davidson, C. Short Call Poster 41, Short Call Poster 60

Delivanis, D. Short Call Poster 12

Desai, V. Short Call Poster 33

Di Dalmazi, G. Short Call Oral 1

Dietz, A. Short Call Poster 12

DiStefano, J. Short Call Poster 59

Dorsaint, P. Short Call Poster 66

Elcheparova, S. Short Call Poster 67

Elemento, O. Short Call Poster 66

Elisei, R. Short Call Oral 2

Endo, M. Short Call Poster 31

Evans, D.B. 462, Short Call Poster 55

Evans, L. Short Call Poster 21

Fagin, J. Short Call Poster 62 Falcone, R. Short Call Oral 10 Fatima, H. Short Call Poster 52 Fazeli, S. Short Call Poster 45 Fedorov, E. Short Call Poster 67 Ferretti, E. Short Call Oral 2 Folkestad, L. Short Call Oral 6 Frädrich, C. Short Call Poster 28 Franco, A. Short Call Poster 46 Frietze, S. Short Call Poster 41, Short Call Poster 70

Fu, G. Short Call Poster 37

Gandhi, M. Short Call Poster 12

Gao, Q. Short Call Poster 29

Gao, Y. Short Call Poster 11

Gao, Z. Short Call Poster 50

Ge, M. Short Call Poster 39
Ge, X. Short Call Poster 39

Geiger, J. 432, Short Call Poster 20

Gillis, N.E. Short Call Poster 41, Short Call Poster 60, Short Call Poster 70

Goehmann, J. Short Call Poster 28

Goepfert, R. Short Call Oral 9, Short Call Poster 25

Gong, R. Short Call Poster 23

Gorskaya, N. Short Call Poster 67

Goswami, M. Short Call Poster 25

Gross, N. Short Call Oral 9, Short Call Poster 25

Guan, H. 124, 258, Short Call Poster 13

Guan, Q. Oral 38, Short Call Poster 42

Guan, W. Short Call Oral 8

Gule-Monroe, M.K. Short Call Poster 45

Guo, X. Short Call Poster 11

Gustafson, M. Short Call Poster 12

Habra, M.A. 394, Oral 32, Short Call Poster 45

Hai, T. Oral 32, Short Call Poster 40

Hallas, J. Short Call Oral 6

Han, B. Short Call Poster 22, Short Call Poster 35

Han, L. 42, 109, Short Call Poster 18

Han, S. Short Call Poster 73, Short Call Poster 75

Hanlon, B. Short Call Poster 69

Hao, X. Short Call Poster 22

Hao, Y. 461, 462, Short Call Poster 55

Hasbay, E. Short Call Poster 52

Hasbellaoui, F. Short Call Poster 19

Hegedus, L. Short Call Oral 6

Henderson, B. Short Call Poster 56, Short Call Poster 57

Henderson, J. Short Call Poster 56

Henriksen, D. Short Call Oral 6

Ho, J. Short Call Oral 5

Ho, T. Short Call Poster 33

Hoffman, M. Short Call Oral 9, Short Call Poster 40, Short Call Poster 45 Högqvist Tabor, M. Short Call Poster 57

Högqvist Tabor, V. Short Call Poster 57

Hou, P. Short Call Poster 13, Short Call Poster 16, Short Call Poster 17, Short Call

Hu, M.I. 76, 394, 462, Oral 32, Short Call Oral 10, Short Call Poster 45, Short Call Poster 55

Huang, X. Short Call Poster 33

Ippolito, S. Short Call Oral 1

Isaza, A. Short Call Poster 46

Jhiang, S.M. Short Call Poster 38

Ji, q. 230, Short Call Poster 42 
Jiang, Y. Short Call Poster 23

Jimenez, C. 355, 394, Oral 32, Short Call Oral 10, Short Call Poster 45

Jin, C. Short Call Poster 30

Jonklaas, J. 47, 398, Oral 25, Oral 5, Short Call Poster 59

Joshi, N. Short Call Poster 20

Kabadi, U.M. Short Call Poster 36

Kachergus, J. Short Call Oral 3

Kandil, E. 118, 154, 175, 179, 182, 183, 263, 324, 377, 382, 409, 467, Short Call Poster 32

Karelina, J. Short Call Poster 67

Kargi, A. Short Call Poster 73, Short Call Poster 75

Kaya, C. Oral 39, Short Call Poster 66

Kazahaya, K. Short Call Poster 46

Kennedy, G.C. 461, 462, Short Call Poster 55

Kilgore, P. Short Call Poster 61

Kim, C. 473, Short Call Poster 74

Kim, K. 272, 367, Short Call Poster 71

Kloos, R.T. 461, 462, Short Call Poster 55

Kobaly, K. Short Call Poster 74

Köhrle, J. Oral 6, Short Call Oral 4, Short Call Poster 28

Kottschade, L. Short Call Poster 12

Kotwal, A. 275, Short Call Poster 12

Krane, J.F. 462, Short Call Poster 55

Kuker, R. 303, Short Call Poster 73, Short Call Poster 75

Labourier, E. Short Call Poster 46

Lai, S. 353, Short Call Oral 9, Short Call Poster 25

Lam, L. Short Call Poster 20

Lan, X. Short Call Poster 39

Langer, J. Short Call Poster 74

Lepe, M. Short Call Poster 48

Li, D. Short Call Poster 62

Li, H. Short Call Poster 33

Li, L. 77, 253, Short Call Poster 30

Li, M. Short Call Poster 30

Li, T. Short Call Poster 11

Li, X. 119, Short Call Poster 13, Short Call Poster 16, Short Call Poster 17, Short Call Poster 43

Li, Z. 110, 148, 149, 371, 372, Oral 21, Short Call Poster 23, Short Call Poster 72

Liang, J. Short Call Oral 8

Liao, T. 42, 109, Short Call Poster 18, Short Call Poster 42

Lim, A. Short Call Oral 5

Lim, K. Short Call Poster 33

Lim, M. Short Call Poster 33

Lin, Y. 250, 253, 429, Short Call Oral 8, Short Call Poster 17, Short Call Poster 47, Short Call Poster 50

Liu, C. 333, 334, 383, 454, Short Call Poster 17

Liu, J. Short Call Poster 11

Liu, R. Short Call Poster 32

Liu, W. 77, Short Call Poster 42
Liu, Y. 54, 119, 249, 269, 414, 429, Short Call Oral 8, Short Call Poster 23, Short Call 47

Liu, Z. Short Call Poster 17, Short Call Poster 22, Short Call Poster 68

Livhits, M.J. 57, 326, 462, Short Call Poster 55

Loh, K. Short Call Poster 33

Long, C. Short Call Poster 31

Long, K.L. Short Call Poster 69

Lou, C. Short Call Poster 50

Lu, G. Short Call Poster 11

Lum, J. Short Call Poster 33

lund, L. Short Call Oral 6

Luo, H. 115, Short Call Poster 23, Short Call Poster 72

Luying, G. Short Call Poster 26, Short Call Poster 27

Ma, B. Oral 31, Oral 38, Short Call Poster 18, Short Call Poster 42

Ma, L. Short Call Poster 58

Ma, Y. Short Call Oral 3

Macerola, E. Short Call Oral 7

Mackay, R. Short Call Poster 63, Short Call Poster 64

MacMillan, C. Short Call Poster 37

Mahdavian, E. Short Call Poster 64

Maia, A.L. Short Call Poster 51

Makarin, V. Short Call Poster 67

Malugov, Y. Short Call Poster 67

Mandel, S. 398, Short Call Poster 74

Maniakas, A. Short Call Oral 9, Short Call Poster 25

Marka, N. Short Call Poster 69

Masucci, R. Short Call Poster 24

Mathur, A. Short Call Poster 49

McBeath, E. Short Call Poster 40

McDow, A.D. Short Call Poster 69

Mehta, D. 313, Short Call Poster 54

Menq, C. Short Call Poster 38

Mercurio, S. Short Call Poster 66

Miao, L.Y. Short Call Poster 14, Short Call Poster 15

Mimouni, S. Short Call Poster 19

Molinaro, E. Short Call Oral 2

Monette, S. Short Call Poster 62

Mostoufi-Moab, S. Short Call Poster 46

Munir, M.A. Short Call Poster 49

Nabhan, F. 430, Short Call Poster 31, Short Call Poster 38

Nasr, C. 403, 432, Short Call Poster 20

Nga, M. Short Call Poster 33

Ngiam, K. Short Call Poster 33

Nikiforov, Y.E. 225, 226, 375, 391, Oral 39, Short Call Poster 33, Short Call Poster 48

Nikiforova, M. 225, 226, 375, 391 , Short Call Poster 48, Short Call Poster 66

Ning, Y. Short Call Poster 30

Norman, J. Short Call Poster 65

Novaes, K.F. Short Call Poster 51

Novokshonov, K. Short Call Poster 67

Ocampo, J. Short Call Poster 73, Short Call Poster 75
Okoh, A. Short Call Poster 54

Oliveira, T.S. Short Call Poster 51

Ortiga-Carvalho, T.M. Short Call Poster 51

Ouyang, W. Short Call Poster 50

Pani, F. Short Call Oral 1

Parameswaran, R. Short Call Poster 33

Pellegriti, G. Short Call Poster 24

Peralta, C. Short Call Poster 52

Pilgrim, E. Short Call Poster 56

Pitt, S.C. Short Call Poster 69

Poma, A.M. Short Call Oral 7

Porter, k. Short Call Poster 31

Prete, A. Short Call Oral 2

Proietti, A. Short Call Oral 7

R.M, S. Short Call Poster 33

Ramesh, P.C. Short Call Poster 61, Short Call Poster 64

Ramone, T. Short Call Oral 2

Rao, A.D. Short Call Poster 33

Rao, N. Short Call Poster 33

Razavi, C.R. Short Call Poster 32

Razavi, M.A. 377, Short Call Poster 32

Renko, K. Short Call Oral 4, Short Call Poster 28

Ridouani, F. Short Call Poster 62

Rijntjes, E. Short Call Oral 4

Ringel, M. Short Call Poster 40

Rivas, A. Short Call Oral 3

Rivera, M. Short Call Oral 3

Rocco, P.R. Short Call Poster 51

Roll, K. 430, Short Call Poster 31

Romei, C. Short Call Oral 2

Rosvall, B.R. Short Call Poster 34

Roy, R. Short Call Poster 65

Ruan, B. Short Call Poster 43

Russell, J. 407, 409, Short Call Poster 32

Russomanno, G. Short Call Oral 7

Ryder, M. 396, Short Call Poster 12

Sabini, E. Short Call Oral 1

Sablin, I. Short Call Poster 67

Sadow, P. 462, Short Call Poster 55

Sapuppo, G. 232, Short Call Poster 24

Saucke, M.C. Short Call Poster 69

Sboner, A. Short Call Poster 66

Schmid, K. Short Call Oral 4

Schweizer, U. Short Call Oral 4

Scortegagna, E. Short Call Poster 73, Short Call Poster 75

Segev, D. Short Call Poster 49

Sek, K.S. Short Call Poster 33

Semenov, A. Short Call Poster 67

Seth, A. 313, Short Call Poster 54

Shaear, M. 407, 409, Short Call Poster 32

Shah, M. 430, Short Call Poster 40

Shaheen, S.A. Short Call Poster 52

Shalaby, M. 154, 175, 182, 183, 263, 324, 377, 409, Short Call Poster 32

Sherman, S.I. 304, 394, 462, Oral 32, Short Call Oral 10, Short Call Poster 45 , Short Call Poster 55

Shi, B. 59, 113, 185, 328, Oral 14, Oral 19, Short Call Poster 16, Short Call Poster 43 
Silva, P.L. Short Call Poster 51

Singh, B. Short Call Oral 5

Sinha, R. Short Call Oral 5

Sipos, J.A. 461, Short Call Poster 31

Sleptsov, I. Short Call Poster 67

Smallridge, R.C. Short Call Oral 3

Solomon, S. Short Call Poster 62

Song, Y. 112, 120, 270, Short Call Poster 30

Sorisky, A. Short Call Poster 58

Sperling, J. Short Call Oral 9,

Short Call Poster 25

Spranger, J. Short Call Oral 4

Stack, B.C. 462, Short Call Poster 55

Stratton, C. Short Call Poster 64

Sturgis, E. Short Call Oral 9, Short Call Poster 25

Su, P. Short Call Poster 22

Sui, F. Short Call Poster 13, Short Call Poster 16,

Short Call Poster 43

Suleman, A. Short Call Poster 52

Surrey, L. Short Call Poster 46

Sutton, W. Short Call Poster 49

Tacito, A. Short Call Oral 2

Tai, E. Short Call Poster 33

Tai, Z. Short Call Poster 39

Tan, A. Short Call Poster 33

Tan, J. 244, 253, 257, Short Call Poster 50

Tan, S. Short Call Poster 33

Tan, W. Short Call Poster 33

Tan, Z. 291, Short Call Poster 39

Tang, J. 148, 149, Short Call Poster 53

Tavarelli, M. Short Call Poster 24

Tessler, F. Short Call Poster 76

Thompson, A. Short Call Oral 3

Timofeeva, N. Short Call Poster 67

Tolino, L. 375, Short Call Poster 33, Short Call Poster 48

Tomczak, J. Short Call Poster 41, Short Call Poster 60, Short Call Poster 70

Torregrossa, L. 335, Short Call Oral 2

Tufano, R.P. 407, 409, Short Call Poster 32
Tun, T. Short Call Poster 33

Tuttle, R. Short Call Poster 62

Ugolini, C. Short Call Oral 2

Uspenskaya, A. Short Call Poster 67

Viola, D. Short Call Oral 2

Waguespack, S. 76, Oral 32, Short Call Oral 10, Short Call Poster 45

Wajner, S. Short Call Poster 51

Wald, A.I. Short Call Poster 33, Short Call Poster 48

Wang, C. 161, 228, Short Call Oral 8, Short Call Poster 39

Wang, J. 59, 124, 162, 185, 258, 291, 415, Short Call Oral 9, Short Call Poster 25, Short Call Poster 39

Wang, L. Oral 15, Short Call Poster 23, Short Call Poster 72

Wang, R. 212, 253, Short Call Poster 21, Short Call Poster 50

wang, s. Short Call Poster 30

Wang, X. 119, 269, 450, Oral 31, Short Call Oral 3

Wang, Y. 59, 111, 227, 269, 328, Oral 19, Oral 28, Oral 31, Oral 38, Short Call Poster Wei, Q. Short Call Poster 30

Wei, T. Short Call Poster 23

Wei, W. Oral 38, Short Call Poster 39

Wei, Z. Short Call Poster 50

Weinberger, P. Short Call Poster 61, Short Call Poster 63, Short Call Poster 64

Weitzman, S. Short Call Oral 10

Wen, D. Oral 31, Short Call Poster 42

Wha Eng, K. Short Call Poster 66

Wiese, N. Short Call Poster 28

Wirth, E.K. Short Call Oral 4

Witterick, I. Short Call Poster 37

Wong, R. Short Call Poster 62

Wright, C. Short Call Poster 38
Wu, M. 176, Short Call Poster 68

Xi, X. Short Call Poster 26, Short Call Poster 27

Xu, H. 119, 162, 291, Short Call Poster 72

$\mathrm{Xu}$, J. Short Call Poster 39

$\mathrm{Xu}, \mathrm{Q}$. Short Call Poster 61, Short Call Poster 63, Short Call Poster 64

Xu, W. Oral 31, Oral 38, Short Call Poster 18

Yang, N. Short Call Poster 69

Yang, Q. Short Call Poster 13, Short Call Poster 16, Short Call Poster 43

Yang, S. 119, 325, Oral 38, Short Call Poster 33

Yang, T. Short Call Poster 23

Yen, P.M. Short Call Oral 5

Young, A. Short Call Poster 21

Yu, Y. Short Call Poster 11

Zafereo, M.E. 394, Oral 32, Short Call Oral 9, Short Call Poster 25

Zayouna, C. Short Call Poster 20

Zeiger, M. 111, Oral 28, Short Call Poster 49

Zhang, B. 162, Short Call Poster 29, Short Call Poster 53

Zhang, C. 54, Short Call Poster 39

Zhang, H. 44, Short Call Poster 22

Zhang, J. Short Call Poster 11

Zhang, L. 48, 49, 347, 407, Short Call Poster 23

Zhang, Q. 77, Short Call Poster 39

Zhang, R. Short Call Poster 50

Zhang, S. 132, 353, Short Call Poster 72

Zhang, X. 44, 249, 333, 334 , Short Call Oral 8, Short Call Poster 47 Zhao, C. 119, Short Call Poster 11 Zhen, J. Short Call Poster 58 Zheng, C. 291, Short Call Poster 39 Zhou, J. 244, 271, Short Call Oral 5 Zhu, J. 271, 433, Short Call Poster 23 Zhu, X. Short Call Poster 39 